12 Azarisman MS, Fauzi MA, Faizal MP, et al. The SAFE (SGRQ score, air-flow limitation and exercise tolerance) Index: a new composite score for the stratification of severity in chronic obstructive pulmonary disease. Postgrad Med J 2007; 83: 492-497.

13 Cardoso F, Tufanin AT, Colucci M, et al. Replacement of the 6-min walk test with maximal oxygen consumption in the BODE Index applied to patients with COPD: an equivalency study. Chest 2007; 132: 477-482.

14 Schembri S, Anderson W, Morant S, et al. A predictive model of hospitalisation and death from chronic obstructive pulmonary disease. Respir Med 2009; 103: 1461-1467.
15 Esteban C, Arostegui I, Moraza J, et al. Development of a decision tree to assess the severity and prognosis of stable COPD. Eur Respir J 2011; 38: 1294-1300.

16 Altman DG, Vergouwe $Y$, Royston $\mathrm{P}$, et al. Prognosis and prognostic research: validating a prognostic model. BMJ 2009; 338: b605.

17 Moons KG, Altman DG, Vergouwe Y, et al. Prognosis and prognostic research: application and impact of prognostic models in clinical practice. BMJ 2009; 338: b606.

DOI: 10.1183/09031936.00088011

\title{
Avoiding backward steps in COPD: looking again at
} \section{roflumilast}

\section{To the Editors:}

I read with interest the article by BATEMAN et al. [1] on the influence of roflumilast when it is used concomitantly with longacting $\beta_{2}$-agonists to reduce exacerbations. Similarly, roflumilast remained effective with concomitant short-acting muscarinic antagonists. Phosphodiesterase (PDE) 4 inhibitors have arisen as anti-inflammatory drugs that may prevent exacerbations in moderate to severe chronic obstructive pulmonary disease (COPD) patients who fulfil the chronic bronchitis profile. Preclinical investigations suggest that roflumilast mitigates tobacco smoke-induced lung inflammation, mucociliary malfunction, lung fibrotic remodelling, oxidative stress or pulmonary vascular remodelling. However, clinical studies have failed to identify inflammation biomarkers that demonstrate significant and evident anti-inflammatory effects of roflumilast.

There are two potential controversies from the basic research. First, COPD may be understood from the point of view of PDE4 dysfunction, which may explain systemic COPD manifestations through mechanisms alternative to those solely of inflammation. PDE4 has been implicated in fetal survival and growth, control of airway smooth muscle tone, adaptation velocity of contraction in cardiac myocytes, mood regulation, neutrophil recruitment, and promotion of the tumour necrosis factor (TNF)- $\alpha$ inflammation pathway. Knockout models and pharmacological inhibition of PDE4 have also demonstrated antidepressant effects and triggers for arrhythmias or heart failure. All these findings have led to the study of the role of PDE4 in heart failure, cardiovascular risk, sarcopenia, osteoporosis, anaemia and depression. Interestingly, these are associated comorbidities currently described as extrapulmonary manifestations of COPD [2]. Conversely, PDE4 mutations and polymorphisms, initially described in neuropsychiatric disorders or ischaemic stroke, have also been recently encountered in COPD or osteopenia. Homma et al. [3] investigated the association between the polymorphisms of the PDE4D gene and the development of COPD. They found significant differences between the COPD and control groups in the distribution of SNP13 allele frequencies of the PDE4D gene and haplotypes composed of PDE4D SNP7 and IL13 +2044 G/A. This may suggest that a group of COPD patients corresponds to PDE4 altered phenotypes whose systemic manifestations may improve in response to roflumilast.
Secondly, roflumilast may act not as an anti-inflammatory drug but as a bronchodilator with additional systemic effects through PDE inhibition. When the clinical trials were analysed, compared with all other treatments, the combination of roflumilast plus long-acting muscarinic antagonist exhibited the largest treatment effects [4]. This is predictable, taking into account this inhibition of airway hyperreactivity to muscarinic cholinergic agonists. Cyclic nucleotides play a critical role in the control of smooth muscle relaxation by intersecting the $\mathrm{Ca}^{2+}$-signalling pathway that promotes contraction at several critical points. When a mouse model of allergic asthma was compared with wild-type littermates, PDE4D knockout mice sensitised intraperitoneally and challenged intranasally with ovoalbumin do not exhibit airway hyperreactivity to the muscarinic cholinergic agonist methacholine [5]. The effect of PDE4 ablation appears to be specific for muscarinic cholinergic stimulation because it is possible to induce contraction by other constrictors, such as serotonin or vasopressin. In fact, the primary end-point of the tiotropium plus roflumilast trial (M2-128 study) was change in forced expiratory volume in $1 \mathrm{~s}$, which was demonstrated.

In conclusion, it would be a serious backward step from the previous advances on COPD and PDE inhibitors if new and on-going investigations only focus on airways, and do not consider clinical evidence of inflammation. None of the clinical trials are long enough to test results on inflammation processes. Likewise, they do not investigate inflammation and compare biomarkers between roflumilast and placebo groups. It is uncertain whether roflumilast can stop inflammation in the mild COPD patient in the long term, or whether it will become inefficient after a 1-yr treatment in the moderate-tosevere patient. I hypothesise that the group of COPD with systemic manifestations may have PDE4 dysfunction and the response to PDE inhibitors could be clinically and biochemically superior to the other COPD groups. I suggest further investigations with biomarkers, such as those of endothelial dysfunction (P-selectin, intercellular adhesion molecule (ICAM)-1, ICAM-3, vascular cell adhesion molecule-1 and Eselectin) and adipokines (apolipoprotein A1, apolipoprotein B, lipoprotein A and paraoxonase), which are closely associated with the inflammatory process observed in patients with other inflammatory chronic diseases that follow the TNF- $\alpha$ pathway. 
Equally, it would be interesting to explore whether roflumilast responders show PDE4 polymorphisms.

\section{L.M. Blasco}

UARH, Hospital Universitario Marqués de Valdecilla, Santander, Spain.

Correspondence: L.M. Blasco, UARH, Pab. 13, -1, Hospital Marqués de Valdecilla, Avenida Marqués de Valdecilla, 39008, Santander (Cantabria), Spain. E-mail: grullus99@yahoo.es

Statement of Interest: None declared.

\section{REFERENCES}

1 Bateman ED, Rabe KF, Calverley PM, et al. Roflumilast with longacting $\beta_{2}$-agonists for COPD: influence of exacerbation history. Eur Respir J 2011; 38: 553-560.

2 Barnes PJ, Celli BR. Systemic manifestations and comorbidities of COPD. Eur Respir J 2009; 33: 1165-1185.

3 Homma S, Sakamoto T, Hegab AE, et al. Association of phosphodiesterase 4D gene polymorphisms with chronic obstructive pulmonary disease: relationship to interleukin 13 gene polymorphism. Int J Mol Med 2006; 18: 933-939.

4 Mills EJ, Druyts E, Ghement I, et al. Pharmacotherapies for chronic obstructive pulmonary disease: a multiple treatment comparison meta-analysis. Clin Epidemiol 2011; 3: 107-129.

5 Hansen G, Jin S, Umetsu DT, et al. Absence of muscarinic cholinergic airway responses in mice deficient in the cyclic nucleotide phosphodiesterase PDE4D. Proc Natl Acad Sci USA 2000; 97: 6751-6756.

DOI: $10.1183 / 09031936.00140311$

\section{From the authors:}

We thank L.M. Blasco for his comments on our article, and for drawing attention to the potential broader effects of phosphodiesterase (PDE) inhibitors in chronic obstructive pulmonary disease (COPD) and its comorbidities [1]. The end-points in our report were limited to those necessary for obtaining registration of roflumilast for use in COPD. However, we agree that it would be unfortunate if future studies focused only on the airways, as this class of drug has potential for addressing several other mechanisms and comorbidities in COPD.

L.M. Blasco challenges the notion that the effects of roflumilast in COPD are the result of an anti-inflammatory effect of the drug. He suggests that these might result from bronchodilation and systemic PDE4 inhibition. We disagree with this explanation for several reasons. First, although he is correct that increased levels of cyclic adenosine monophosphate have smooth muscle relaxant effects, both clinical and in vitro studies of the role of selective PDE4 inhibitors, such as roflumilast, have failed to confirm an acute bronchodilator effect [2-4]. These studies include the use of cilomilast in COPD [2] and roflumilast in asthma [3]. Secondly, as L.M. Blasco acknowledges, several preclinical studies have shown that roflumilast targets inflammatory processes in COPD, with beneficial effects on tobacco-induced lung inflammation and emphysema, bleomycin-induced lung fibrosis, and mucociliary malfunction and oxidative stress [5], effects that are likely to be highly relevant in COPD. In a 4-week, placebo-controlled crossover study in patients with COPD, roflumilast treatment resulted in reductions in the absolute number of neutrophils, lymphocytes, macrophages and eosinophils, soluble interleukin- 8 , neutrophil elastase, eosinophilic cationic protein, and $\alpha_{2}$-macroglobulin in sputum [6]. While we agree that this observation requires confirmation in a prospective lung biopsy study of COPD patients treated with roflumilast, a previously reported study performed with cilomilast administered for 12 weeks confirmed a reduction in inflammatory cell numbers in bronchial biopsy specimens [7]. Therefore, our current hypothesis is that the improvements in lung function and exacerbation reductions seen with roflumilast are most likely the result of anti-inflammatory activity.

Finally, L.M. Blasco refers to the work of HommA et al. [8] in Japanese patients, which explores the association between PDE4 polymorphisms and the development of COPD. He speculates that these patients might benefit more from PDE4 inhibition. However, it must be noted that a functional consequence of these polymorphisms on PDE4 activity has not yet been demonstrated. To date, upregulation of PDE4 activity has been reported only in alveolar macrophages of smokers with moderate COPD [9] and is attributed to chronic inflammation and the oxidative stress found in COPD. We agree that more research is required to understand the relationship between levels of PDE4 activity in COPD and sustained PDE4 inhibition.

\section{E.D. Bateman* , K.F. Rabe, ${ }^{\#, ~, ~ P . M . A . ~ C a l v e r l e y ~}{ }^{+}$, U.M. Goehring ${ }^{\S}$, M. Brose ${ }^{f}$, D. Bredenbröker ${ }^{\S}$ and L. Fabbri**}

*Pulmonology Division, Dept of Medicine, University of Cape Town, Cape Town, South Africa. "Dept of Medicine, University of Kiel, Kiel, "Krankenhaus Grosshansdorf, Center for Pulmonology and Thoracic Surgery, Grosshansdorf, ${ }^{\S}$ Respiratory Medicine, Nycomed GmbH, and ${ }^{f}$ Data Science, Nycomed GmbH, Konstanz, Germany. ${ }^{+}$School of Clinical Sciences, University Hospital Aintree, Liverpool, UK. ${ }^{* *}$ Dept of Oncology, Haematology and Respiratory Diseases, University of Modena and Reggio Emilia, Modena, Italy.

Correspondence: E.D. Bateman, University of Cape Town Lung Institute, George Street, Mowbray 7700, Cape Town, South Africa. E-mail: Eric.Bateman@uct.ac.za

Statement of Interest: Statements of interest for all authors and for the study itself can be found at www.erj.ersjournals.com/ site/misc/statements.xhtml

\section{REFERENCES}

1 Bateman ED, Rabe KF, Calverley PMA, et al. Roflumilast with longacting $\beta_{2}$-agonists for COPD: influence of exacerbation history. Eur Respir J 2011; 38: 553-560.

2 Grootendorst DC, Gauw SA, Baan R, et al. Does a single dose of the phosphodiesterase 4 inhibitor, cilomilast (15 mg), induce bronchodilation in patients with chronic obstructive pulmonary disease? Pulm Pharmacol Ther 2003; 16: 115-120.

3 Engelstatter R, Wingertzahn M, Schmid-Wirlitsch C, et al. Roflumilast, an oral once-daily phosphodiesterase 4 (PDE4) inhibitor does not exhibit bronchodilator activity. Ann Allergy Asthma Immunol 2005; 94: 169. 